\title{
Detailed dynamics of electron beams self-trapped and accelerated in a self-modulated laser wakefield
}

\author{
S.-Y. Chen, M. Krishnan, A. Maksimchuk, R. Wagner, and D. Umstadter \\ Center for Ultrafast Optical Science, University of Michigan, Ann Arbor, Michigan 48109
}

(Received 5 April 1999; accepted 9 September 1999)

\begin{abstract}
The electron beam generated in a self-modulated laser-wakefield accelerator is characterized in detail. A transverse normalized emittance of $0.06 \pi \mathrm{mm}$ mrad, the lowest ever for an electron injector, was measured for $2 \mathrm{MeV}$ electrons. The electron beam was observed to have a multicomponent beam profile and energy distribution. The latter also undergoes discrete transitions as the laser power or plasma density is varied. In addition, dark spots that form regular modes were observed in the electron beam profile. These features are explained by analysis and test particle simulations of electron dynamics during acceleration in a three-dimensional plasma wakefield.
\end{abstract} (C) 1999 American Institute of Physics. [S1070-664X(99)03612-5]

\section{INTRODUCTION}

Acceleration of electrons by an electron plasma wave has been of great current interest because of its much larger (four orders of magnitude) acceleration gradient than that of conventional $\mathrm{rf}$ linacs $(<20 \mathrm{MeV} / \mathrm{m}) .{ }^{1}$ Several methods have been proposed for driving a large-amplitude fast-phasevelocity plasma wave, ${ }^{1}$ such as the plasma wakefield accelerator, the plasma beat-wave accelerator, the Laser WakeField Accelerator (LWFA), the resonant laser plasma accelerator, and the Self-Modulated Laser Wake-Field Accelerator (SMLWFA). The former two methods were demonstrated first because the required technologies, e.g., relativistic electron beam or long-pulse medium-power laser, were well developed. The LWFA and the SMLWFA have recently received considerable attention and shown tremendous progress because of invention of ultrashort-duration terawatt-peak-power lasers based on the chirped-pulse amplification technique. ${ }^{2}$

In the LWFA, an electron plasma wave is driven resonantly by a short laser pulse through the laser ponderomotive force. ${ }^{3}$ In the SMLWFA, an electron plasma wave is excited by a relatively long laser pulse undergoing the stimulated Raman forward scattering instability. ${ }^{4-6}$ The injection of electrons can occur uncontrollably by trapping of hot background electrons, which are preheated by other processes such as Raman backscattering and sidescattering instabilities, ${ }^{7-9}$ or by wave breaking (longitudinal ${ }^{1}$ or transverse ${ }^{10}$ ). It can also be achieved by specific injection schemes $^{11,12}$ in order to control the characteristics of the generated electron beam. In this case, self-trapping is also important because the electrons accelerated by it represent a dark current, which may ultimately limit the maximum plasma-wave amplitude that can be used in a plasma-based accelerator, and that also forms a noise source for applications of the electron beam. An understanding of the dynamics of electron trapping and detrapping during acceleration in a plasma wave is fundamental to the design of viable plasma-based accelerators.

Several groups have observed the generation of $\mathrm{MeV}$ electrons from the SMLWFA. ${ }^{8,9,13-15}$ A two-temperature distribution in the electron energy spectrum was reported by Malka et al. ${ }^{15}$ They attributed such a distribution to be a result of the combination of two different acceleration mechanisms, i.e., acceleration by a laser field and by a plasma wave. Gordon et al. ${ }^{16}$ have observed the acceleration of electrons beyond the linear dephasing limit, and explained it, using Particle-In-Cell (PIC) simulations, as a result of acceleration in wakefields driven by accelerated electrons. $\mathrm{We}^{14}$ have previously observed that the generated electron beam has a two-component spatial beam profile, and that the temperature of electrons in the low-energy range undergoes an abrupt change, coinciding with the onset of extension of the laser channel due to self-guiding of the laser pulse, when the laser power or plasma density is varied. Several PIC and test particle simulations ${ }^{7,17-21}$ have also been done to study the characteristics of the electron beam accelerated in a plasma wave, in addition to simple theoretical analyses given in, e.g., Refs. 21-23. However, none of these experiments has revealed the dynamics of electron acceleration in an electron plasma wave, and little direct comparison between the theories (simulations) and the experiments has been made.

In this experiment, the electron beam produced from a self-modulated laser wakefield accelerator, injected with self-trapping of electrons, was characterized in detail. The observations of up-to-three-component electron-beam profiles and up-to-two discrete changes in the slope of electron energy distribution are reported. In addition, dark spots that form regular modes were observed in the first beam-profile component. These new observations provide us important new clues to the underlying dynamics of electron acceleration in a three-dimensional (3-D) plasma wave. The observed phenomena could be explained by use of a 3-D test particle simulation, which is based on a simple model that takes into account only the longitudinal and transverse electric fields of the electron plasma wave and trapping of electrons along the entire plasma-wave channel. In this paper we present a study of dynamics of electron acceleration in a plasma wave for the first time, via the comparison between the experimental re- 


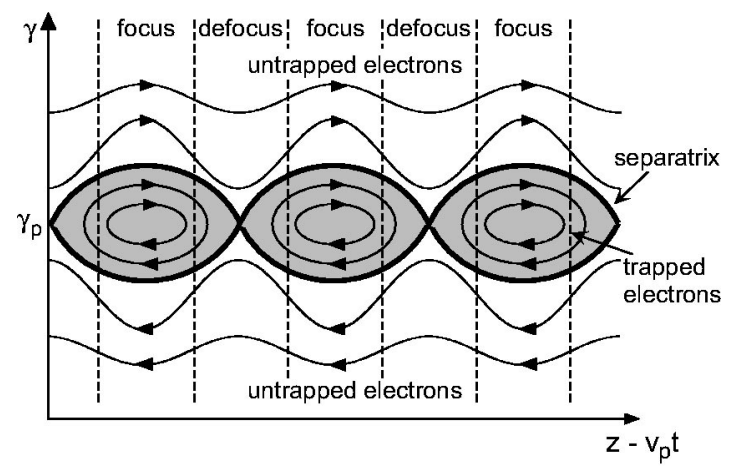

FIG. 1. Phase-space trajectories for electrons in a plasma wave. Electrons above and below the separatrix are untrapped, while electrons within the separatrix (shaded) are trapped by the wave. The motion of the electrons relative to the wave is indicated by the arrows. The separatrix is symmetric with respect to $\gamma_{p}$, when the $\gamma$ axis is in a logarithmic scale. Vertical dashed lines separate transversely focusing and defocusing regions that result from the transverse electric field.

sults and simulations. In addition, an electron beam with a normalized emittance that is an order of magnitude lower than that of best rf guns was produced. This shows that a laser wakefield accelerator can potentially serve as a better injector because the high acceleration gradient can lower the beam emittance by minimizing the time during which electrons are nonrelativistic and thus susceptible to space charge effects. $^{11}$

This paper is organized as follows. In Sec. II, basic dynamics of electron acceleration in a one-dimensional (1-D) electron plasma wave is reviewed. In Sec. III, diagnostic systems used for characterization of the electron beam are shown, and the results of the experiment are presented. In Sec. IV, a simple 3-D test particle simulation code was used to analyze the features of electron acceleration in a 3-D plasma wave. The results are compared with the experimental observations. A summary is given in Sec. V.

\section{BASIC PHYSICS OF LASER-PLASMA-BASED ELECTRON ACCELERATORS}

\section{A. Motion of electrons in a 1-D plasma wave}

Trapping and acceleration of a test electron in a nonlinear plasma wave were analyzed in one dimension using Hamiltonian dynamics by Esarey and Pilloff. ${ }^{23}$ The motion of electrons in the plasma wave can be represented by a phase-space diagram (Fig. 1), which shows the orbits of the electrons in the plasma wave. Electrons inside the bounded region (inside the separatrix) are "trapped" by the plasma wave and carried along in the same wavelength of the plasma wave (often called a 'bucket'). Electrons above and below this bounded region are "untrapped.' When an electron is below the separatrix initially, it gains and loses energy quickly, and never has an energy higher than that corresponding to the phase velocity of the plasma wave. On the other hand, when an electron is inside the separatrix, it can move to the top of the separatrix and gain significant energy. This is the process of electron acceleration in a plasma wave. As a result, the bottom and the top of the separatrix deter- mine the minimum trapping threshold and the maximum electron energy attainable, respectively, for a given plasmawave amplitude. They are given by

$$
\begin{aligned}
& \gamma_{\min }=\gamma_{p}\left(1+\gamma_{p} \Delta \phi\right)-\gamma_{p} \beta_{p}\left[\left(1+\gamma_{p} \Delta \phi\right)^{2}-1\right]^{1 / 2}, \\
& \gamma_{\max }=\gamma_{p}\left(1+\gamma_{p} \Delta \phi\right)+\gamma_{p} \beta_{p}\left[\left(1+\gamma_{p} \Delta \phi\right)^{2}-1\right]^{1 / 2},
\end{aligned}
$$

where

$$
\Delta \phi=2 \beta_{p}\left[\left(1+\epsilon^{2} / 2\right)^{2}-1\right]^{1 / 2},
$$

in which $\gamma_{p}=1 / \sqrt{1-\beta_{p}^{2}}$ is the relativistic factor of plasmawave phase velocity, $\beta_{p}=\mathrm{v}_{p} / c$ is the normalized phase velocity, $\epsilon=E_{\max } / E_{b}$ is the plasma-wave amplitude, and $E_{b}$ $=m_{e} \omega_{p} \mathrm{v}_{p} / e$ is the nonrelativistic cold wave-breaking limit. The actual trapping threshold and the actual maximum energy for each electron depends on its position (phase) in the plasma wave at injection.

The maximum electron energy attainable in a plasma wave increases with an increase of the plasma-wave amplitude, which is limited by wave breaking. The latter is defined by the point at which the plasma wave traps the bulk of electrons that constitute the plasma wave itself and thus selfdestructs. In a cold plasma, the maximum plasma-wave amplitude is given by ${ }^{24}$

$$
E_{\text {max }}=E_{0} \sqrt{2\left(\gamma_{p}-1\right)},
$$

and the maximum electron energy is ${ }^{23}$

$$
\gamma_{\max }=4 \gamma_{p}^{3}-3 \gamma_{p} .
$$

For a higher plasma temperature, the wave-breaking limit is lowered, due to trapping of hot bulk electrons at a lower plasma-wave amplitude. ${ }^{25,26}$ For a plasma wave with an amplitude below the wave-breaking limit, it can trap hot electrons that are at the tail of a Maxwellian distribution of a thermal plasma, or that are preheated to exceed the trapping threshold by other mechanisms, or that are injected externally. In this case, these trapped electrons are accelerated and thus take energy away from the plasma wave, resulting in damping of the plasma wave. This is referred to as electron beam loading or nonlinear Landau damping. ${ }^{27-29}$ In fact, untrapped electrons can also gain energy (as seen in Fig. 1) and damp the wave, a process referred to as Landau damping. ${ }^{30}$

\section{B. Limitations on laser-plasma-based electron accelerators}

Under practical conditions, in a plasma wave of a certain amplitude, the maximum energy gain for an electron is limited by the acceleration distance. Three main factors determine the acceleration distance. The first one is the dephasing between the electron and the plasma wave. Electrons are accelerated when they are in the accelerating region of a plasma wave. Because of the increase of the electron energy with propagation distance, the electrons can outrun the plasma wave after a certain distance, enter the decelerating region of the plasma wave and start to lose energy. This maximum acceleration distance is referred to as the electron detuning length, and is given by $L_{d} \simeq \gamma_{p}^{2} \lambda_{p}$ in the 1-D case, where $\lambda_{p}$ is the plasma-wave wavelength. 
The other two factors that can result in a shorter acceleration distance and thus a lower-energy gain are the diffraction limit and the pump depletion limit. These two limits determine the actual length of the plasma-wave channel excited by a laser pulse. In order to drive a large-amplitude plasma wave, usually the laser pulse has to be focused down to a small spot in order to achieve a high laser intensity. However, the natural diffraction of the laser beam results in a finite length over which the high laser intensity can be maintained. This length is the Rayleigh length, which, e.g., is only $220 \mu \mathrm{m}$ for a $10 \mu \mathrm{m}$ focal spot and $1 \mu \mathrm{m}$ wavelength. This limit can be overcome by guiding of the laser pulse in the plasma through self-guiding or preformed-plasmachannel guiding. ${ }^{1,31}$ The pump depletion limit $L_{\mathrm{pd}}$ is usually less stringent. ${ }^{32-36}$ It is due to the conversion of laser energy to the plasma wave, and thus can be calculated by equating the energy in the laser pulse with the energy in the plasma wave for the case of LWFA, resulting in $L_{\mathrm{pd}}$ $=c \tau \gamma_{p}^{2}\left(a_{0} / \epsilon\right)^{2}$, where $\tau$ is the laser pulse duration and $a_{0}$ is the amplitude of the normalized vector potential of the laser field. However, such an estimate is inappropriate for SMLWFA, because, in this case, depletion of the laser energy might be dominated by other strong loss mechanisms such as Raman sidescattering and backscattering. ${ }^{37}$ Another limitation for the maximum electron energy is determined by electron beam loading, in which the plasma-wave amplitude decreases while the electrons gain energy. The latter two factors determine the conversion efficiency of laser energy to the total electron energy.

\section{CHARACTERISTICS OF THE GENERATED ELECTRON BEAM}

The experiment was performed with a Ti:sapphire/ $\mathrm{Nd}$ :glass laser system that produced 400 fs duration laser pulses at $1.053 \mu \mathrm{m}$ wavelength with a maximum peak power of $4 \mathrm{TW}$. The $50 \mathrm{~mm}$ diam laser beam was focused with an $f / 3.3$ parabolic mirror onto the front edge of a supersonic helium gas jet. The focal spot in vacuum was a $7 \mu \mathrm{m}$ FWHM (full width at half-maximum) near-Gaussian spot (which contained $60 \%$ of the total pulse energy) and a large dim spot (100 $\mu \mathrm{m}$ FWHM). The helium gas was fully ionized by the foot of the laser pulse. At a laser power of $\geqslant 2 \mathrm{TW}$ and a plasma density of $\geqslant 2 \times 10^{19} \mathrm{~cm}^{-3}$, the laser pulse underwent relativistic-ponderomotive self-channeling, ${ }^{14,31}$ and the laser channel extended to be $750 \mu \mathrm{m}$ in length, the length of the gas jet. The length and the diameter of the laser channel were monitored by side imaging of Thomson scattering of the laser pulse propagating in the plasma.

Under such conditions, an electron plasma wave was excited by the laser pulse through stimulated Raman forward scattering instability, as was evident from the observation of Raman satellites in the spectrum of the transmitted light. ${ }^{8}$ An electron beam was produced, when the laser power or gas density exceeded a certain threshold, and propagated in the direction of the laser beam. The spatially averaged timeresolved plasma-wave amplitude was measured using colinear collective Thomson scattering. ${ }^{38}$ The peak plasma-wave amplitude was observed to increase with increase of laser

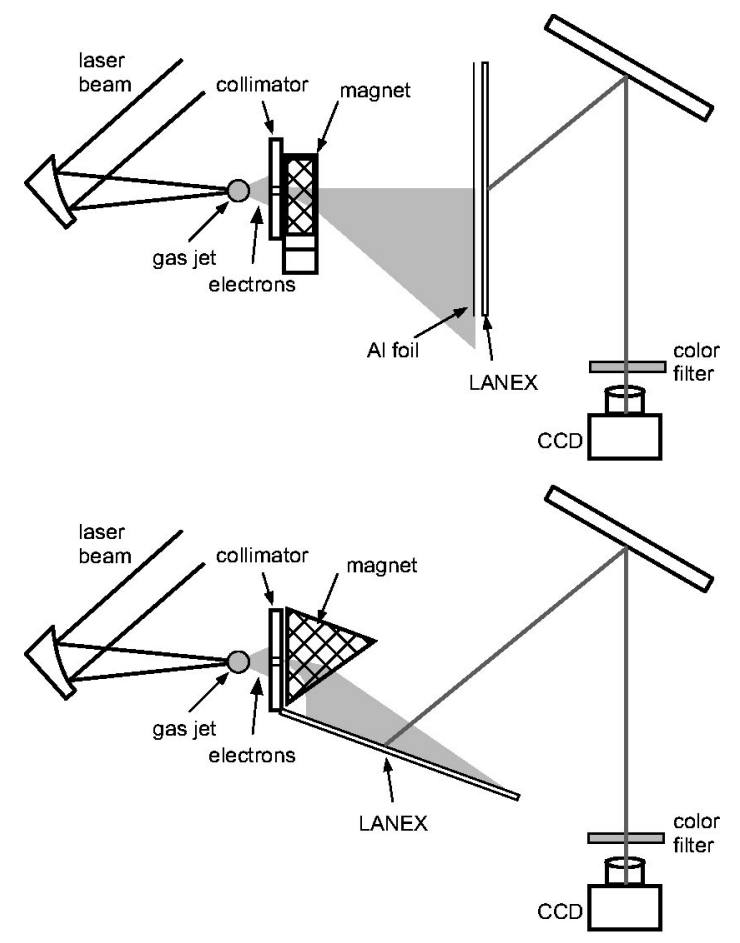

FIG. 2. Diagrams of the setups for measuring electron energy spectra in the low-energy range. In the upper diagram, the dispersion of electrons on the LANEX plane is nonlinear. In the lower diagram, the dispersion is linear, and the LANEX is on the point-to-point imaging plane of the sector magnet.

power or plasma density, and the maximum observed was about $\epsilon=0.3$. The generated electron beam can be characterized by its energy distribution (which determines the longitudinal emittance), its beam divergence (which determines the transverse emittance) and its total number of electrons.

\section{A. Diagnosis of the electron beam}

The electron energy spectrum in the low-energy range $(<8 \mathrm{MeV})$ was measured using a dipole permanent magnet with a KODAK LANEX scintillating screen imaged by a CCD (charge-coupled device) camera as the detector. A rectangular dipole magnet or a sector dipole magnet was used, as shown in Fig. 2. The results obtained using these two different setups are found to be identical within the error bar. A collimator with an $f / 30$ cone angle was put in front of the magnet to select electrons propagating in a specific direction and to obtain a high momentum resolution. Higher-energy electron energy spectra were obtained by using dipole electromagnets, a multiwire proportional chamber (MWPC), and a collimator with an $f / 100$ cone angle, as shown in Fig. 3.

The electron-beam spatial profile at $16 \mathrm{~cm}$ away from the gas jet was measured using a LANEX screen imaged by a CCD camera, as that shown in the upper diagram of Fig. 2 with the collimator and the magnet removed. Because of the aluminum foil in front of the LANEX, which was used for blocking the laser light, and the back support of the LANEX, only electrons with kinetic energies higher than $100 \mathrm{keV}$ were imaged. Since the source size of the generated electron beam was small, $\sim 10 \mu \mathrm{m}$ in diameter and $<750 \mu \mathrm{m}$ in length (as determined by the diameter and the length of the laser channel), the electron beam profile on the LANEX was 


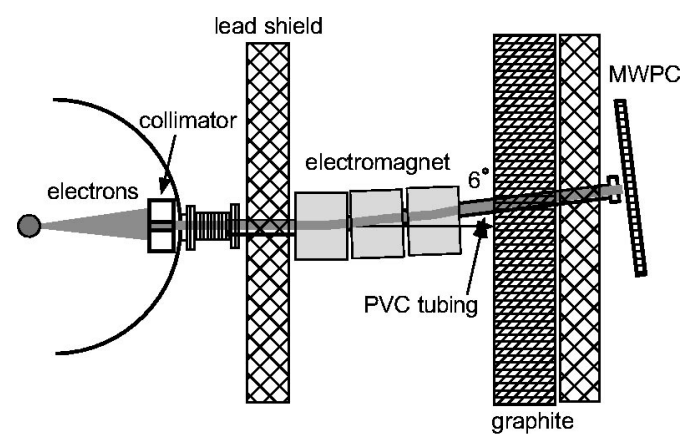

FIG. 3. Diagram of the setup for measuring electron energy spectra in the high-energy range. By scanning the strength of the magnetic field of the magnets while monitoring the MWPC readings, the electron energy spectrum is obtained.

actually a measurement of the electron beam divergence (angular pattern).

The total number of electrons in the beam was measured using two different methods. The first one is by using a Faraday cup to directly collect the entire electron beam and measure the total charge on a storage oscilloscope. The other measurement of the total electron number was done by using a collimator and a calibrated scintillator-PMT (photomultiplier tube). This calibrates the relation between the LANEX emission intensity and the absolute electron flux. By integrating over the whole electron beam image on the LANEX, the total number of electrons was obtained. The difference between these two measurements was within a factor of 2 . Such a difference was actually dominated by fluctuations of the electron beam itself.

\section{B. Number of electrons}

The total number of electrons in the generated electron beam was measured at various laser powers and plasma densities. ${ }^{8}$ Figure 4 shows the total number of electrons in the beam as a function of laser power. Below a threshold, no electron is observed. When the laser power exceeds this threshold, the number of electrons increases exponentially

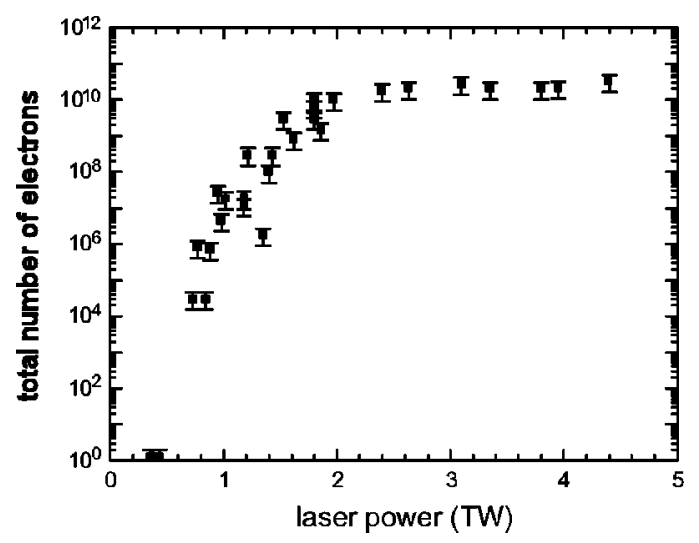

FIG. 4. The total number of electrons in the generated electron beam as a function of laser power at $3.7 \times 10^{19} \mathrm{~cm}^{-3}$ plasma density. Because of a 25 $\mu \mathrm{m}$ thick aluminum foil used for blocking the laser light (in front of the Faraday cup or wrapping the PMT), electrons with an energy below $60 \mathrm{keV}$ was not measured.
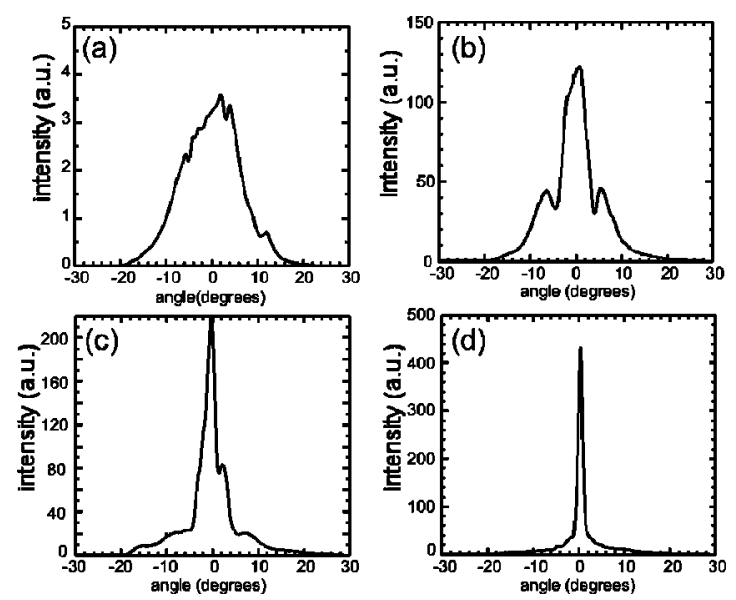

FIG. 5. Lineouts of the electron beam profiles for various laser powers at $2.3 \times 10^{19} \mathrm{~cm}^{-3}$ plasma density: (a) $0.6 \mathrm{TW}$, (b) $1.1 \mathrm{TW}$, (c) $2.0 \mathrm{TW}$, and (d) $2.9 \mathrm{TW}$.

with an increase of laser power, and then gradually saturates to a certain level. The threshold of the electron production is believed to be determined by the electron trapping threshold. When the laser power is increased, the trapping threshold becomes lower because of the increase of the plasma-wave amplitude [see Eq. (1)]. At the same time, the maximum energy of the preheated electrons also increases because of an increase of the amplitude of the slow plasma wave excited through Raman backscattering or because of enhancement of other heating processes. Therefore, the production threshold of the electrons occurs when the maximum energy of the preheated electrons exceeds the trapping threshold. The exponential growth of the number of electrons with laser power is expected from the exponential increase of the number of preheated electrons that are above the trapping threshold. The saturation of the number of electrons at high laser powers may result from the beam loading effect, in which the electrons trapped saturate the plasma wave by removing its energy. The variation of the number of electrons with change of plasma density shows the same behavior for basically the same reasons.

\section{Transverse beam profile}

The electron beam profile (angular pattern) was observed to contain several concentric Gaussian-like-profile beams, and the number of beam components depends on laser power and plasma density. For a plasma density of $2.3-6.2 \times 10^{19} \mathrm{~cm}^{-3}$, only one beam component $\left(\sim 20^{\circ}\right.$ FWHM) exists in the electron beam at $0.6 \mathrm{TW}$ laser power. At a laser power larger than $1 \mathrm{TW}$, a second beam component grows up on top of the first beam component, with a divergence angle of $\sim 7.5^{\circ}$ FWHM. For 2.3-3.4 $\times 10^{19} \mathrm{~cm}^{-3}$ plasma density, a third beam component with a divergence angle of $1^{\circ}-3^{\circ}$ FWHM appears when the laser power is higher than $2 \mathrm{TW}$. The ratio between the peak of the third component and that of the second component increases with increasing laser power and reaches as high as a factor of 10. Figure 5 shows the lineouts of the electron 

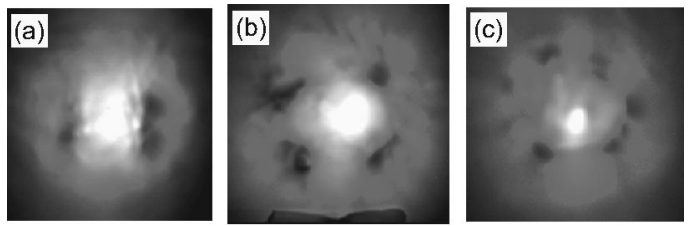

FIG. 6. Images of the transverse electron beam profiles at various laser powers and plasma densities: (a) $1.1 \mathrm{TW}, 3.4 \times 10^{19} \mathrm{~cm}^{-3}$; (b) $3.5 \mathrm{TW}$, $6.2 \times 10^{19} \mathrm{~cm}^{-3}$; and (c) $2.0 \mathrm{TW}, 2.3 \times 10^{19} \mathrm{~cm}^{-3}$.

beam profiles for various laser powers at $2.3 \times 10^{19}-\mathrm{cm}^{-3}$ plasma density.

The divergence angles of the first (widest), second, and third beam components are about $20^{\circ}-25^{\circ}, 5^{\circ}-10^{\circ}$, and $1^{\circ}-3^{\circ}$ FWHM, respectively. Basically, for the second beam component, its divergence angle increases with an increase of laser power. For the third beam component, the divergence angle decreases and its relative intensity (with respect to the first and second beam components) increases with an increase of laser power. However, the divergence angle of the first beam component is roughly invariant with variation of laser power and plasma density. This indicates that the effect of change of plasma-wave amplitude and plasma frequency on the beam divergence angle of the first beam component is negligible. In addition, it implies that the nonlinearity of the plasma wave and the self-generated magnetic field have no effect on the beam divergence angle, because the results are roughly the same for very small and very large plasma-wave amplitudes. Furthermore, it shows that the space charge effect (occurring during and after the acceleration) on the beam divergence is insignificant, because no significant change in the divergence angle is observed even when the number of accelerated electrons varies by several orders of magnitude (note that the divergence resulting from the space charge effect should be roughly proportional to $\rho^{1 / 2}$, where $\rho$ is the charge density of the electron beam). Therefore, it seems that the multicomponent beam profile and its divergence angle are just simply intrinsic properties of electron acceleration in self-consistent longitudinal and transverse electric fields of a plasma wave.

The appearance of the second beam component was observed to roughly coincide with the sudden extension of the plasma-wave channel (which is determined by the laser channel) caused by laser self-guiding. In addition, when the second beam component appears, there are usually some holes appearing in the first beam component, as shown in
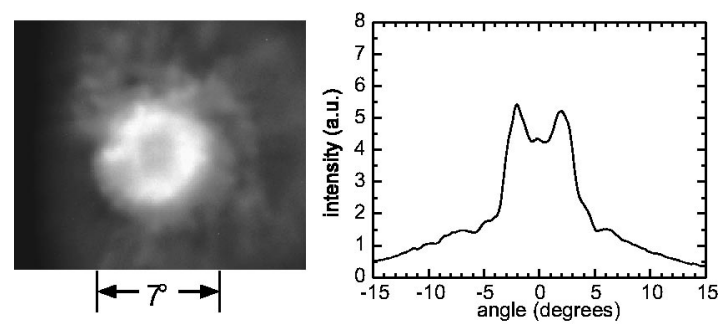

FIG. 7. Image and vertical lineout of the transverse electron beam profile at $3.5 \mathrm{TW}$ laser power and $6.2 \times 10^{19} \mathrm{~cm}^{-3}$ plasma density.

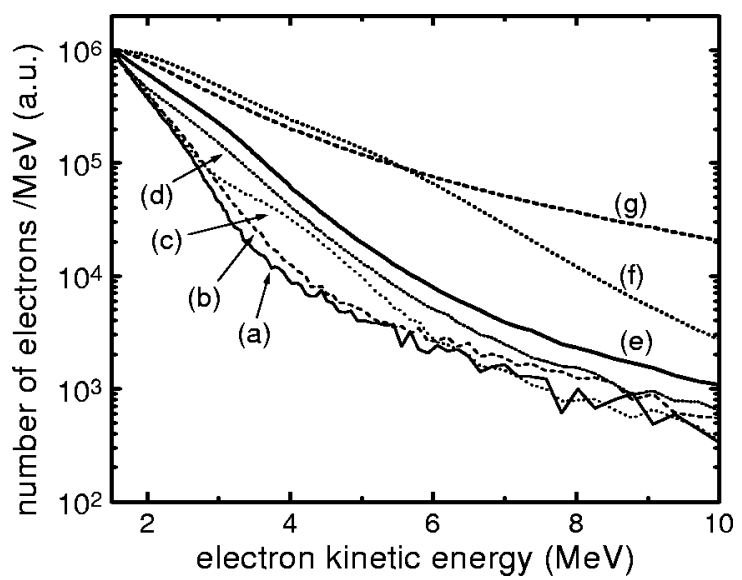

FIG. 8. Electron energy spectra for various laser powers and plasma densities: (a) $2.6 \mathrm{TW}, 3.4 \times 10^{19} \mathrm{~cm}^{-3}$; (b) $2.9 \mathrm{TW}, 3.5 \times 10^{19} \mathrm{~cm}^{-3}$; (c) $3.3 \mathrm{TW}$, $4.8 \times 10^{19} \mathrm{~cm}^{-3}$; (d) $3.9 \mathrm{TW}, 4.8 \times 10^{19} \mathrm{~cm}^{-3}$; (e) $1.7 \mathrm{TW}, 6.2$ $\times 10^{19} \mathrm{~cm}^{-3}$; (f) $2.7 \mathrm{TW}, 6.2 \times 10^{19} \mathrm{~cm}^{-3}$; and (g) $3.5 \mathrm{TW}, 6.2$ $\times 10^{19} \mathrm{~cm}^{-3}$.

Fig. 6. These holes form regular patterns that are similar to $\mathrm{TM}_{12}, \mathrm{TM}_{22}$, and $\mathrm{TM}_{32}$ electromagnetic modes in a circular waveguide, or to $(1,0),(1,1)$, and $(1,2)$ Hermite-Gaussian modes of a laser beam. Furthermore, under the highest laser power and plasma density achievable in this experiment, a density depression at the center of the accelerated electron beam was observed occasionally, as shown in Fig. 7.

\section{Electron energy spectrum}

Figure 8 shows the normalized electron energy spectra in the low-energy range for various laser powers and plasma densities. Figure 9 shows the results taken under a different laser focus condition ${ }^{14}$ (for these data, the gas density is $1.8 \times 10^{19} \mathrm{~cm}^{-3}$ at 1000 psi backing pressure) and Fig. 10 shows the corresponding side images of the laser channel. The spectra were found to have Maxwellian-like distributions, i.e., $\exp (-\alpha \gamma)$, where $\gamma$ is the relativistic factor of electron energy and $\alpha$ is a fitting parameter $[(511 \mathrm{keV}) / \alpha$ is the temperature]. The slope, $\alpha$, of the spectrum was found to change discretely with variation of laser power and plasma density. For instance, at a fixed plasma density, the slope remains the same with increasing laser power until a certain threshold is reached. Then the slope $\alpha$ changes to a lower value, and stays the same with a further increase of laser power until the next jump. The same behavior occurs for varying plasma density at a fixed laser power. Three $\alpha$ values
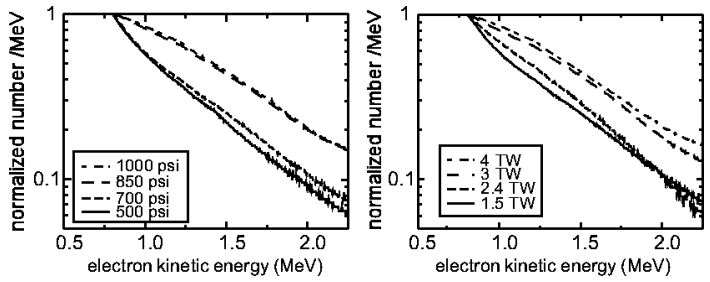

FIG. 9. Electron energy spectra for various gas-jet backing pressures at a fixed laser power of $3 \mathrm{TW}$ (left), and for various laser powers at a fixed plasma density of $3.6 \times 10^{19} \mathrm{~cm}^{-3}$ (right). 
(d)

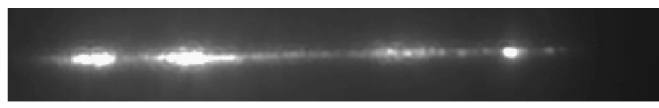

(c)

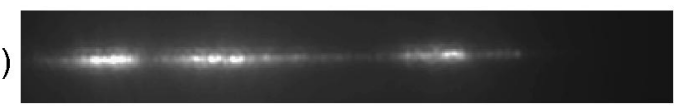

(b)

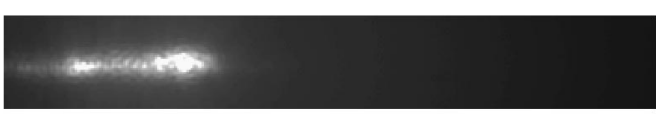

(a)

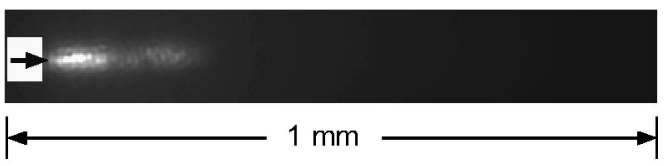

FIG. 10. Side images of the laser channel for various laser powers at a fixed plasma density of $3.6 \times 10^{19} \mathrm{~cm}^{-3}$ : (a) $1.5 \mathrm{TW}$, (b) $2.4 \mathrm{TW}$, (c) $3 \mathrm{TW}$, (d) 4 TW. The arrow indicates the direction of laser propagation.

(two jumps) were observed in this experiment: 1.0, 0.6, and 0.3 . The occurrence of the first jump roughly coincides with the extension of the laser channel from $<400$ to $750 \mu \mathrm{m}$. However, when the second jump occurs, there is no change in the laser channel length (it already reaches the maximum length limited by the gas jet). Following this trend, further jumps may be possible if the laser power or plasma density can be increased further. For some rare conditions, the energy spectrum seems to be a mixture of two different slopes, such as line (c) in Fig. 8. By changing the positions of the collimator and the magnet to look at the spectra of electrons ejected in different angles, the slopes of the electron energy spectra in the low energy range were found to be the same for all three beam components, and thus the discrete changes of slopes occur simultaneously for all of them.

Another important observation in this experiment is a two-temperature distribution in the electron energy spectrum. As shown in Fig. 11, which was obtained using both the low-energy and high-energy electron spectrometers, the slope of electron energy distribution in the low-energy range $(\leqslant 5 \mathrm{MeV})$ is steep, while the slope in the high-energy range is much less steep (almost flat). Such a two-temperature distribution was also observed in Fig. 8 and further verified by using aluminum absorbers of various thicknesses in front of

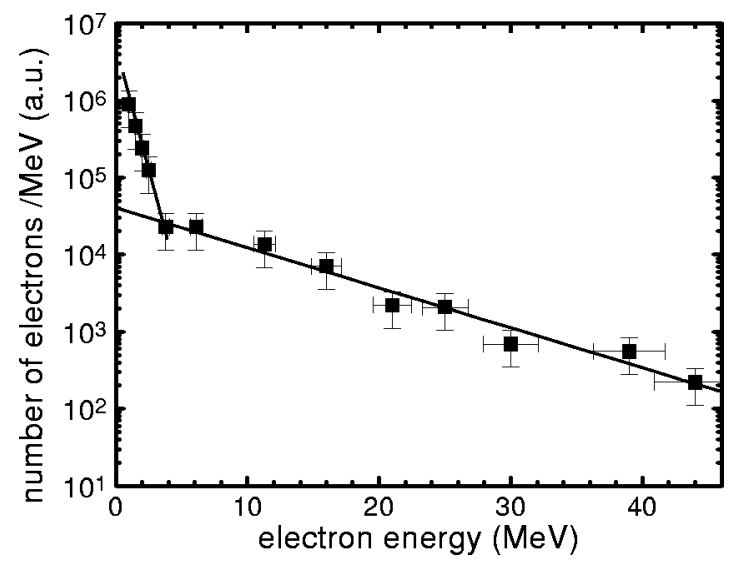

FIG. 11. Electron energy spectrum for 3 TW laser power and 3 $\times 10^{19} \mathrm{~cm}^{-3}$ plasma density. The solid lines represent exponential fits. an electron detector. We cannot identify the high-energy cutoff of the spectrum because of the low signal-to-noise ratio in the high-energy end. However, electrons with energies higher than $40 \mathrm{MeV}$ were observed.

\section{E. Other parameters}

The other two parameters of the electron beam, which are required in order to fully characterize the beam, are the temporal duration of the electron bunch and the transverse cross section of the beam at the source. The temporal duration of the electron pulse should be roughly equal to that of the plasma wave, since the former is generated from the latter. Therefore, the temporal duration of a macrobunch should be about 2 ps, equal to the measured duration of the plasma wave, as discussed in Ref. 38. The separation between adjacent microbunches is equal to the plasma-wave period, which is 18 fs for a plasma density of 3.4 $\times 10^{19} \mathrm{~cm}^{-3}$. The duration of a microbunch should be less than that. Therefore, the maximum peak current is estimated to be about $1 \mathrm{kA}$ or higher. However, this estimation is correct only when the electron pulse is at or near the source. As the electron pulse propagates, its duration becomes larger and larger because of its $100 \%$ energy spread. The large energy spread, the changing duration, and the low repetition rate make a direct measurement of the electron pulse duration difficult.

The transverse cross section of the electron source should be roughly equal to the cross section of the plasma wave, which roughly equals that of the laser beam. It is estimated to be about $10 \mu \mathrm{m}$. In the best cases, the angular divergence of the electron beam was measured to be $\sim 1^{\circ}$, which leads to a normalized transverse emittance of 0.06 $\pi \mathrm{mmmrad}$ for $2 \mathrm{MeV}$ electrons and a brightness ( $B$ $=I /\left[\left(\pi \Delta r^{2}\right)\left(\pi \Delta \theta^{2}\right)\right]$, where $I$ is the beam current, $\Delta r$ is the beam radius, and $\Delta \theta$ is the beam divergence angle) of $8 \times 10^{4} \mathrm{~A} / \mathrm{mm}^{2} \mathrm{mrad}^{2}$. This shows a promising advantage over a conventional rf linac, which has a normalized transverse emittance of $\sim 1 \pi \mathrm{mm}$ mrad at best. ${ }^{39-41}$

\section{DYNAMICS OF ACCELERATION OF ELECTRONS BY A 3-D PLASMA WAVE}

\section{A. Model and basic kinetics}

To understand the physical origin of these phenomena, a simple 3-D test particle simulation code was run and its results were compared with the experimental observations. In this simulation, monoenergetic electrons (with a longitudinal kinetic energy $T_{e z}$ in the direction of the phase velocity of the plasma wave) are injected into predefined and selfconsistent longitudinal and transverse electric fields of an electron plasma wave. The injected electrons also have small (e.g., $400 \mathrm{eV}$ ) transverse momenta initially with random orientations. (The value of the initial transverse momentum does not affect the results in any obvious way as long as it is much smaller than the longitudinal momentum.) The magnetic field is neglected in this simulation and the transverse electric field is derived from the longitudinal field by $\partial E_{r} / \partial z=\partial E_{z} / \partial r$, which results from Maxwell's equations 

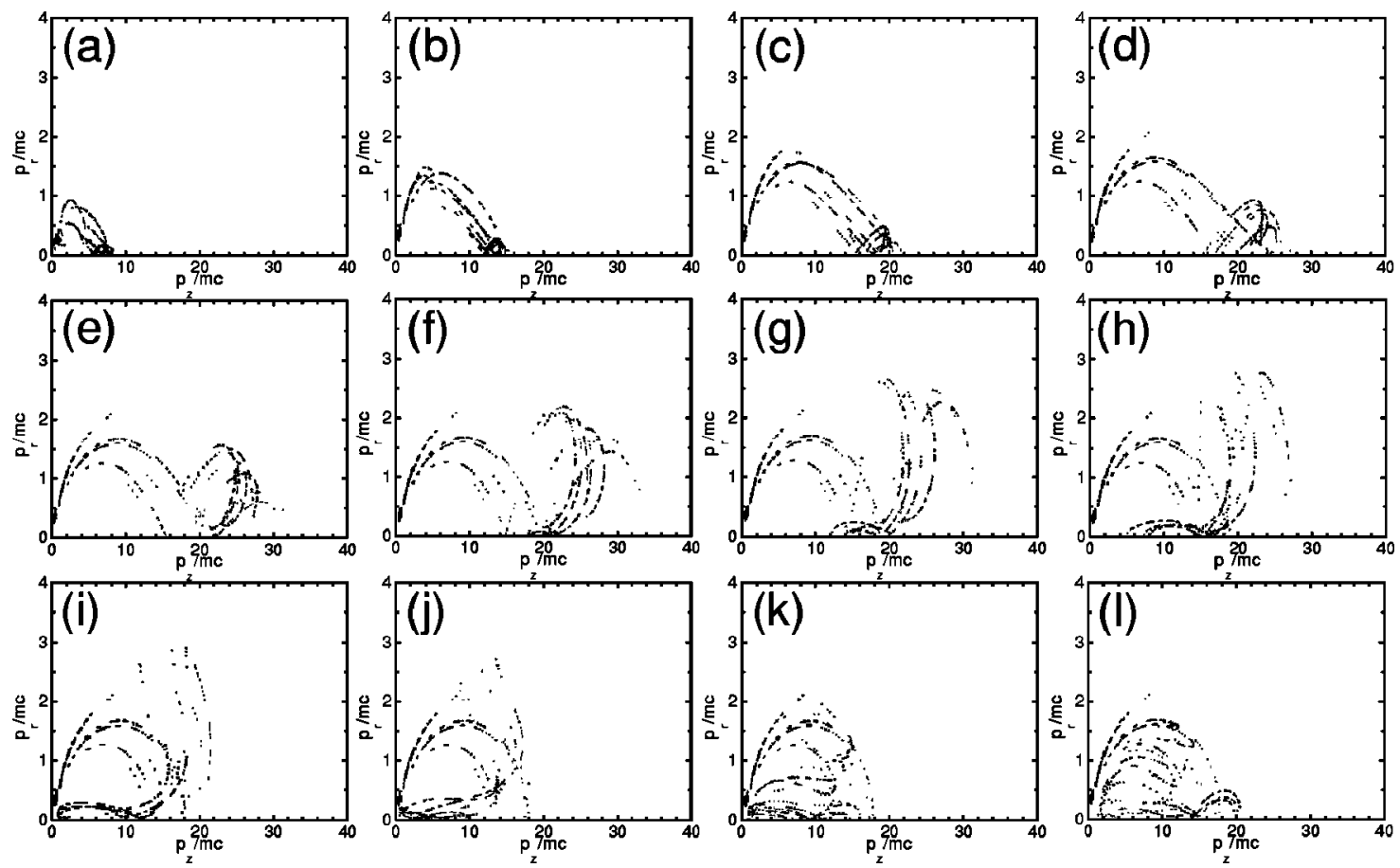

FIG. 12. Simulations of the momentum distributions of electrons injected in one plasma period after propagating various distances for $\epsilon=0.3, r_{0}=5 \mu \mathrm{m}$, $\omega_{p}=3.4 \times 10^{14} \mathrm{rad} / \mathrm{s}$, and $T_{e z}=200 \mathrm{keV}$ : (a) 22, (b) 44, (c) 65, (d) 87, (e) 109, (f) 131, (g) 152, (h) 174, (i) 196, (j) 218, (k) 240, and (l) 261 $\mu \mathrm{m}$.

with $\mathbf{B}$ equaling zero or a constant. (In fact, from the Panofsky-Wenzel theorem, ${ }^{42}$ even when a consistent magnetic field is taken into account, such a relation still holds for a relativistic particle except that $E_{r}$ and $E_{z}$ are then effective electric fields that take into account the effect of the magnetic field as well.) The electric field that is assumed is

$$
\begin{aligned}
\vec{E}(r, \phi, z)= & \hat{z} E_{0} \exp \left(-r^{2} / r_{0}^{2}\right) \cos \left(k_{p} z-\omega_{p} t\right) \\
& +\hat{r} E_{0} k_{p}^{-1}\left(-2 r / r_{0}^{2}\right) \exp \left(-r^{2} / r_{0}^{2}\right) \\
& \cdot \cos \left(k_{p} z-\omega_{p} t-\pi / 2\right),
\end{aligned}
$$

where $r_{0}$ is the radius of the plasma wave, $E_{0}$ is the peak longitudinal electric field, $k_{p}$ is the wave number of the plasma wave, and $\omega_{p}$ is the plasma frequency. At first, 1800 electrons are injected uniformly into a region of $r_{0}(x)$ $\times r_{0}(y) \times \lambda_{p}(z)$ at the beginning of the plasma-wave channel to study the trajectories of the electrons in the 3-D phase space. The space-charge forces between the injected electrons and the effects of these electrons on the plasma wave (the beam loading effect) are neglected.

Figure 12 shows the evolution of the momentum distribution of electrons injected in one plasma-wave bucket for $\epsilon=0.3, r_{0}=5 \mu \mathrm{m}, \omega_{p}=3.4 \times 10^{14} \mathrm{rad} / \mathrm{s}$, and $T_{e z}=200 \mathrm{keV}$. After the injection, electrons that are not trapped inside the separatrix are expelled by the transverse field outward and their momenta fall onto an ellipsoidal contour. The trapped electrons are mainly confined near $p_{r}=0$ and move toward higher $p_{z}$ (higher energy) with time. When they reach the maximum energy (the upper limit of the separatrix) after propagating one electron-detuning-length, $L_{d} \simeq f \gamma_{p}^{2} \lambda_{p}$, where $f\left(=\frac{1}{2}-1\right)$ depends on $r_{0} / \lambda_{p}$, the electrons turn back and move toward the decreasing $p_{z}$ direction (lower energy).
After the electrons reach the lower limit of the separatrix (the trapping threshold), they turn again and move toward higher $p_{z}$, and so on.

While the trapped electrons move in an oscillatory trajectory inside the separatrix (with a bounce period of $2 L_{d} / c$ ), they also drag a tail that spreads in the region confined by the ellipsoidal contour, as a result of the transverse defocusing field of the plasma wave. When the electrons fall onto the contour surface, it means that they have exited the region of the plasma wave transversely. The main process for loss (detrapping) of electrons occurs whenever electrons slow down to enter the defocusing region after passing through the top of the separatrix (a focusing region), as a result of the excessive transverse momenta they obtained in the top focusing region. Therefore, fewer and fewer electrons are left inside the plasma-wave channel, as they oscillate inside the separatrix. The propagating distance required for any specific electron to lose its confinement is about a little longer than $0,2 L_{d}, 4 L_{d}$, etc., depending on its initial spatial position in the plasma wave (i.e., its position in the 3-D phase space), the plasma-wave amplitude, and the injection energy. The confinement time is longer if the electron is at a position of stronger acceleration and smaller $r$ initially. According to the $p_{r} / p_{z}$ of every electron at any time, the electrons can be divided into three groups. The first group of electrons is distributed over the whole region confined by the ellipsoidal contour. The second group is confined along the $p_{z}$ axis (that is, $p_{r} / p_{z}$ is small). The third group is the electrons that obtain large transverse momenta when they transit from the defocusing region to the focusing region during the acceleration phase. Such momentum blowup is accompanied by shrinkage of the transverse spatial spread of the electrons, 
as a result of the conservation of normalized transverse emittance.

The contour observed in the simulation results from the conservation of energy and momentum for the acceleration of an electron by a plasma wave. Starting from the force equation and the energy equation, after simple algebra, a constant of motion is derived, i.e.,

$$
\gamma-\beta_{p} u_{z}+\phi=\eta \quad(\text { a constant }),
$$

where $\gamma$ is the relativistic factor of electron energy, $\beta_{p}$ is the normalized phase velocity of the plasma wave, $u_{z}=p_{z} / m c$ is the normalized longitudinal momentum of the electron, and $\phi=q \Phi / m c^{2}$ is the normalized potential of the electron in the plasma wave. For an electron that is at rest $\left(\gamma=1, u_{z}=0\right)$ before arrival of the plasma wave $(\phi=0), \eta=1$. Otherwise, $\eta=\left(\gamma-\beta_{p} u_{z}+\phi\right)_{t=0}$, which depends on the potential and the momentum of the electron at the time of injection. After the electron exits the plasma wave, $\phi=0$; so the electron must have a longitudinal momentum and a transverse momentum that satisfy an ellipsoidal equation,

$$
\frac{u_{r}^{2}}{a^{2}}+\frac{\left(u_{z}-d\right)^{2}}{b^{2}}=1 \text {, }
$$

where

$$
\begin{aligned}
& a=\sqrt{\gamma_{p}^{2} \eta^{2}-1}, \\
& b=\gamma_{p} \sqrt{\gamma_{p}^{2} \eta^{2}-1}, \\
& d=\gamma_{p}^{2} \beta_{p} \eta .
\end{aligned}
$$

This equation explains the ellipsoidal contour observed in the simulations. Note that for an electron that is at rest before arrival of the plasma wave with $\gamma_{p}^{2} \gg 1$ and that satisfies $u_{z}$ $\ll \gamma_{p}^{2} \beta_{p}$, the contour can be simplified to a paraboloidal function, $u_{r}^{2} / 2 u_{z}=1$. This paraboloidal contour is identical to the $p_{r}-p_{z}$ relation of electrons accelerated by laser ponderomotive force (direct laser acceleration). ${ }^{43,44}$ Therefore, the appearance of electrons that satisfy the relation $\left(p_{r} / m c\right)^{2} / 2\left(p_{z} / m c\right)=1$ in laser-plasma interactions (e.g., in the simulation of Ref. 19 and the experiment of Ref. 44) does not guarantee that it is a result of direct laser acceleration. It may come from acceleration by plasma waves excited through Raman instability or other mechanisms.

These results can also be applied to the accelerated electrons after they come out of the plasma wave. If the electrons exit the plasma-wave column adiabatically (e.g., when they drift out of the column in the transverse direction slowly), they all fall on the ellipsoidal contour derived from the use of $\phi=0$. If the electrons exit the plasma-wave column nonadiabatically (e.g., when they leave at the end of the column), they fill in this ellipsoid. Therefore, the contour derived with the use of $\phi=0$ gives the boundary of the distribution of accelerated electrons in the phase space, and thus allows us to estimate the maximum divergence angle of the electron beam.

For the case of a self-modulated laser wakefield with self-trapping of electrons, the electrons are expected to be injected (self-trapped) over the entire plasma-wave channel. If, in the preceding simulation (injecting electrons in a single
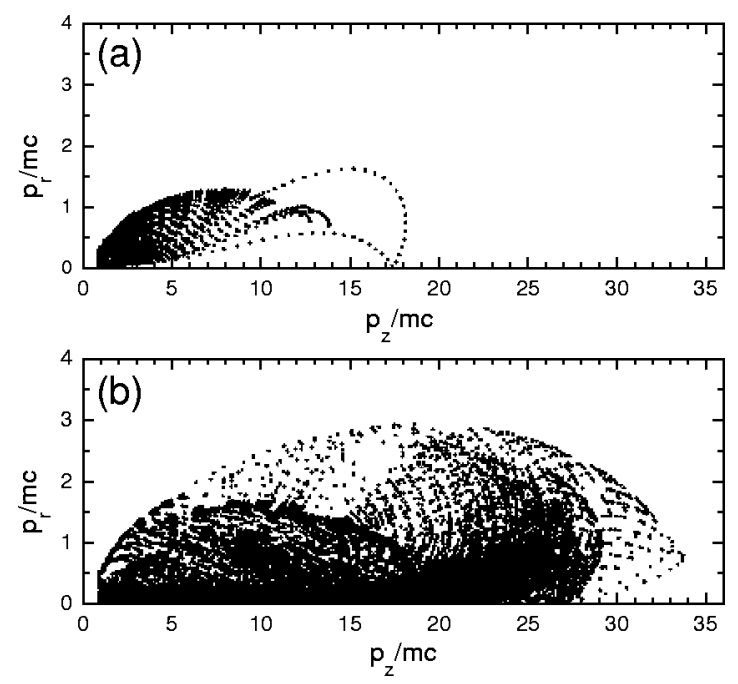

FIG. 13. Simulations of the momentum distributions of electrons injected over the entire $400 \mu \mathrm{m}$ long channel for various plasma-wave amplitudes at $r_{0}=5 \mu \mathrm{m}, \omega_{p}=3.4 \times 10^{14} \mathrm{rad} / \mathrm{s}$, and $T_{e z}=200 \mathrm{keV}$ : (a) $\epsilon=0.15$, and (b) $\epsilon$ $=0.3$. Only the electrons with an energy higher than the injection energy are shown.

bucket), the momentum of each electron is saved at every integer multiple of the plasma-wave period, then a very good approximation of the result for uniform injection over the entire channel can be obtained as a summation of all electrons saved. The results are discussed as follows.

\section{B. Momentum distribution and beam divergence}

The momentum distributions of electrons injected over a plasma-wave channel of $400 \mu \mathrm{m}$ in length are shown in Fig. 13 for various plasma-wave amplitudes. Lineouts of the profiles of the electron beams that correspond to Fig. 13 are shown in Fig. 14. As can be seen clearly, generally three concentric beam components are observed in the electron beam. The first (widest) beam component results from electrons that spread in the whole region confined by the contour. The second and third beam components are composed of electrons distributed in the region near $p_{r}=0$. A ring-like electron beam component may show up under certain conditions, which is a result of a large number of electrons falling onto the contour.

The three beam components observed in the simulations may explain the three-beam-component profile of the experimentally observed electron beam. However, the absolute divergence angles of the three components are not consistent
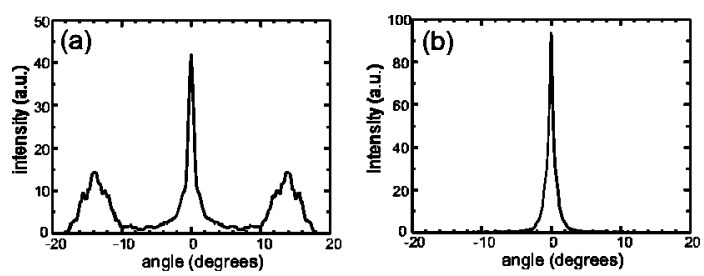

FIG. 14. Simulations of the angular profiles of the electron beams for various plasma-wave amplitudes at $r_{0}=5 \mu \mathrm{m}, \omega_{p}=3.4 \times 10^{14} \mathrm{rad} / \mathrm{s}, T_{e z}$ $=200 \mathrm{keV}$, and $L=400 \mu \mathrm{m}$ : (a) $\epsilon=0.15$, and (b) $\epsilon=0.3$. 


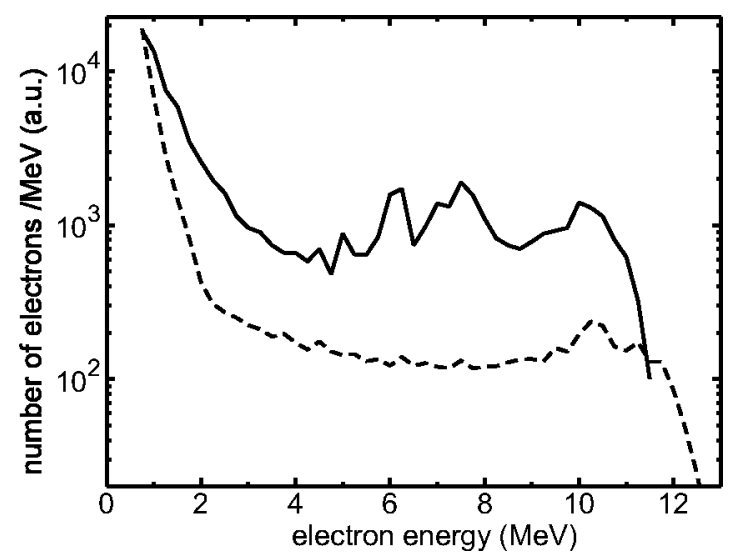

FIG. 15. Simulations of the electron energy spectra for channel radii of $r_{0}$ $=500 \mu \mathrm{m}$ (dashed line) and $r_{0}=5 \mu \mathrm{m}$ (solid line) at $\epsilon=0.15, \omega_{p}=3.4$ $\times 10^{14} \mathrm{rad} / \mathrm{s}$, and $L=400 \mu \mathrm{m}$. The initial energy $T_{e z}$ is distributed randomly between 0 and $300 \mathrm{keV}$. Only the electrons ejected with an angle less than the laser cone angle are counted.

with the experimental results. For the cases shown in Fig. 14, the divergence angle for the first beam component is $12^{\circ}$ FWHM for $\epsilon=0.15$, and $16^{\circ}$ for $\epsilon=0.3$. On the other hand, the experimental result is $\sim 20^{\circ}$ and is roughly invariant with respect to variation of the plasma-wave amplitude or other parameters. This discrepancy is believed to be a result of an inappropriate initial condition $\eta$ used in the simulation. In the simulation, the divergence angle of the first beam component was observed to be roughly proportional to $\eta$, which has a maximum value of $0.59(0.74)$ for the case of $\epsilon$ $=0.15(0.3)$. However, in realistic cases, $\eta$ should be unity for all the electrons because the electrons are initially at rest and the acceleration by a slow plasma wave (for preacceleration) and/or a fast plasma wave should not change $\eta$. Therefore, the divergence angle should be around $21^{\circ}$ by extrapolation and should be invariant with variation of plasma-wave amplitude and plasma frequency. This is quite consistent with the experimental observations.

The results shown in Fig. 13 are very similar to the results of 2-D PIC simulations for self-modulated laser wakefields run by Tzeng et al. ${ }^{19}$ They believed that the electrons that distribute on the $u_{r}^{2} / 2 u_{z}=1$ contour is a result of direct laser acceleration. Nevertheless, our results of the simulations and analyses indicate that the acceleration of electrons by a plasma wave can also lead to such a distribution without considering the effect of the laser field. Therefore, more experimentation is needed in order to discriminate between these two mechanisms.

\section{Energy spectrum}

The electron energy spectra obtained from the simulations show a Maxwellian distribution in the low-energy range, a flat-topped distribution in the high-energy range, and a high-energy cutoff, as shown in Fig. 15. This is consistent with the experimental result. Such a two-temperature distribution also appears in the 1-D simulation, as shown in Fig. 15 by setting $r_{0} / \lambda_{p} \simeq 100$. The exponential distribution in the low-energy range is found to be composed of the untrapped but accelerated electrons (those outside the separa- trix), and electrons that are newly trapped at the end of the channel. (In realistic cases, another contribution for the lowenergy electrons may come from the electrons that are accelerated by a slow plasma wave excited through Raman backscattering or sidescattering.) The energy distribution of the trapped electrons injected in a single bucket is a narrow band with its central energy moving up and down inside the separatrix. In addition, the speed of energy increase with respect to propagating distance is roughly constant, as seen in Fig. 12. In the case of a SMLWFA discussed here, the electrons are injected over the entire channel, and thus the spectrum of the electrons is a summation of all these narrow bands, leading to a flat-topped distribution in the high-energy range.

The high-energy cutoff in the 1-D limit has the same value as in the 1-D analytic result derived by Esarey et al. ${ }^{23}$ for any $\epsilon$, regardless of the sinusoidal wave used in this simulation. However, the high-energy cutoff in the 3-D regime (small $r_{0}$ ) is lower compared to the 1-D result. This is due to the lower $E_{z}$ seen by the electron when it moves to an off-axis position, and this effect is more significant for a larger $\epsilon$. On the other hand, actual experimental measurement might show a cutoff significantly higher than the 1-D theoretical prediction, as a result of the nonlinear correction of the laser group velocity in the plasma (increased by a factor of $\sqrt{\left(\gamma_{\perp}+1\right) / 2}, 45$ where $\gamma_{\perp}$ is the relativistic factor associated with laser intensity) and/or the excitation of a larger-phase-velocity plasma wave driven by the accelerated electron beam (both are not considered in this model). ${ }^{16}$ For instance, under the conditions of Fig. 11 and the measured plasma-wave amplitude of $\epsilon=0.3$, the theoretical highenergy cutoff is calculated to be $32 \mathrm{MeV}$ when Eq. (2) and the nonlinear correction of the laser group velocity are used. This is somewhat lower than the experimental result (40 $\mathrm{MeV}$ or higher). The difference may come from the errors in the measurement of the plasma-wave amplitude or the excitation of plasma waves driven by the accelerated electrons.

Change of injection energy spectrum, plasma wave amplitude, and plasma frequency do not affect the features observed in Fig. 14 and Fig. 15, i.e., the three-component beam profile and the two-slope energy spectrum, except for the ratio between different components.

\section{Transition of the slope of electron energy spectrum}

Prompted by the observation of the simulational results, a possible explanation for the transition of the slope of the electron energy spectrum is given as follows. When the channel length is very short, the energy spectrum is an exponential distribution in the low-energy range. With an increasing channel length, while the slope of energy distribution in the low-energy range remains the same, the energy distribution in the high-energy range becomes a flat-top with its maximum energy extending to a higher energy. The flattopped region reaches an upper limit (the top of the separatrix) when the channel length reaches one electron-detuning length, and then more electrons are added into the flat-topped region toward the lower-energy direction with increasing channel length. At two electron-detuning lengths, as the 


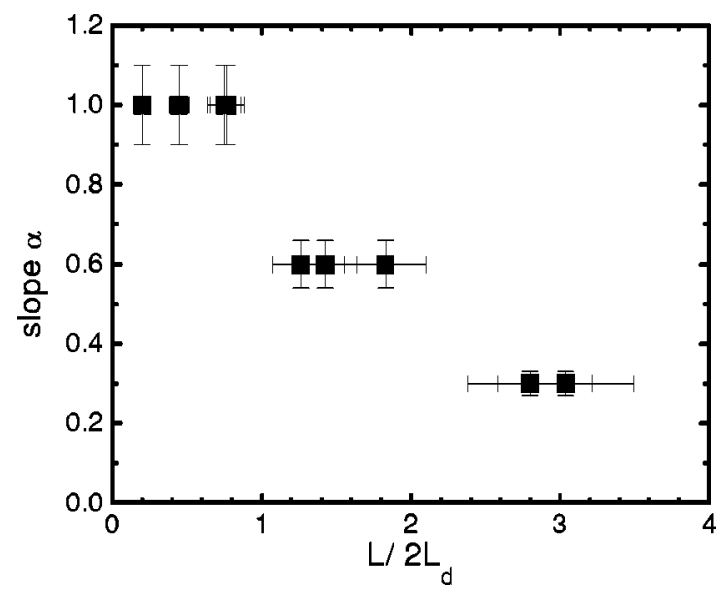

FIG. 16. Slope of the electron energy spectrum in the low-energy range as a function of $L / 2 L_{d}$.

earliest-injected electrons travel back to the bottom (the lowenergy region) of the separatrix, the addition of these electrons to the low-energy spectrum leads to a change in the slope of the exponential distribution. After the channel length is larger than two electron-detuning lengths, the increase of channel length results in an increase of the electron number in the high-energy region once again, and the slope of the energy distribution in the low-energy range stays the same until the next jump, which occurs at four electron detuning lengths.

To compare this with the experimental results, we plot the experimental data on a $\alpha-\left(L / 2 L_{d}\right)$ diagram, as shown in Fig. 16, in which $L$ is the channel length, and $L_{d}\left(\gamma_{p}^{2} \lambda_{p}\right.$ here) is determined from the plasma density. The results show that jumps occur when $L / 2 L_{d}$ is roughly equal to an integer, as expected from above. Qualitatively, an increase of channel length (increasing $L$ ) or increase of plasma density (decreasing $L_{d}$ ) changes $L / 2 L_{d}$ to a larger value, and abrupt changes of the slope are expected to occur at the integer values of $L / 2 L_{d}$. For the cases in which the laser power is increased at a fixed plasma density and a fixed channel length (the length of the gas jet), i.e., fixed $L / 2 L_{d}$, the jumps of the slope can still occur because the confinement time of injected electrons depends on the plasma-wave amplitude. For these cases, $L$ should be replaced by the confinement length, which increases with an increase of the amplitude of the plasma wave (with increasing laser power or plasma density).

\section{E. Dark modes in the beam profile}

There are at least two possible mechanisms that could cause the observed dark-mode structures in the first beam component. The mode structure could be a result of the complicated transverse structure of the plasma wave induced by its nonlinearity at large amplitudes or by self-channeling of the laser pulse. For instance, when self-channeling occurs, the transverse mode of the laser beam might be a higherorder Hermite-Gaussian mode, leading to excitation of an electron plasma wave with a corresponding transverse mode. In simulations for plasma waves assuming such transverse structures, similar mode structures do appear in the first beam component, while the electron beam profile and the energy spectrum still show the same features as in the case for a fundamental Gaussian mode used in Sec. IV. On the other hand, the depression of electron density at the positions of peaks of the transverse laser intensity distribution may lead to a reduction of the plasma wave amplitude at these positions, resulting in dark spots at the corresponding positions in the accelerated electron beam. This may be the cause for the central dark spot shown in Fig. 7.

Other possible causes for the appearance of these dark spots are electron beam instabilities induced by magnetic fields, such as Weibel instability ${ }^{46}$ and Kelvin-Helmholtz instability. ${ }^{47}$ However, these processes cannot be verified in this simulation because space charge forces between the accelerated electrons are not considered.

\section{SUMMARY}

In summary, the characteristics of the electron beam generated from a self-modulated laser wakefield accelerator injected with self-trapping of electrons were measured experimentally, and the main features in the beam profile and the energy spectrum are understood better with the help of simple 3-D test particle simulations that take into account only the longitudinal and transverse electric fields of an electron plasma wave. The multiple-component electron beam profile is believed to be a result of the transverse electric field in a plasma wave. The two-slope electron energy spectrum is found to be a result of electron motion inside and outside the separatrix. The transition of the slope of electron energy spectrum in the low-energy range could be related to the electron detuning length. The dark modes appearing in the electron beam profile may be caused by the excitation of electron plasma waves with higher-order Hermite-Gaussian modes or by density depressions. Furthermore, the results of the simulations and analyses show that electrons that satisfy the relation $\left(p_{r} / m c\right)^{2} / 2\left(p_{z} / m c\right)=1$ may come from ejection by a plasma wave, instead of direct laser acceleration through the laser ponderomotive force.

The experimental observation that there is no obvious dependence of the characteristics of the electron beam on the number of electrons indicates that the space-charge forces between accelerated electrons do not affect the main features of the electron beam. Other factors that could affect the acceleration of electrons in an electron plasma wave include the nonlinearity of the plasma wave and the magnetic field associated with the plasma wave. Since the nonlinear correction of the electric field and the magnitude of the magnetic field ${ }^{48}$ are, in the leading terms, proportional to $\epsilon^{2}$, while the magnitude of the longitudinal and transverse electric fields are proportional to $\epsilon$, these effects can be neglected for small plasma-wave amplitudes $(\epsilon<0.3)$. This is why the qualitative features of the electron beam observed in the experiment can be reproduced in this simplified simulation. For a large plasma-wave amplitude (up to the wave-breaking limit), the nonlinearity and the magnetic field are strong enough to affect the details of the electron acceleration. However, the main features of the electron beam are expected to be about 
the same, because they are simply a result of the conservation of momentum and energy for the acceleration of electrons in a self-consistent plasma wave.

\section{ACKNOWLEDGMENTS}

The electron diagnostic tools were developed in cooperation with D. Gustafson from the Physics Department, University of Michigan, and K. Assamagan and C. Keppel from Hampton University. We would also like to thank E. Dodd, J.-K. Kim, and G. Mourou for their useful discussions. This work was supported by the Division of High Energy Physics, Office of Energy Research, U.S. Department of Energy, and the National Science Foundation.

${ }^{1}$ See references in E. Esarey, P. Sprangle, J. Krall, and A. Ting, IEEE Trans. Plasma Sci. PS-24, 252 (1996).

${ }^{2}$ D. Strickland and G. Mourou, Opt. Commun. 56, 219 (1985).

${ }^{3}$ T. Tajima and J. M. Dawson, Phys. Rev. Lett. 43, 267 (1979).

${ }^{4}$ P. Sprangle, E. Esarey, J. Krall, and G. Joyce, Phys. Rev. Lett. 69, 2200 (1992).

${ }^{5}$ T. M. Antonsen, Jr. and P. Mora, Phys. Rev. Lett. 69, 2204 (1992).

${ }^{6}$ N. E. Andreev, L. M. Gorbunov, V. I. Kirsanov, A. A. Pogosova, and R. R. Ramazashviki, JETP Lett. 55, 571 (1992).

${ }^{7}$ P. Bertrand, A. Ghizzo, S. J. Karttunen, T. J. H. Pättikangas, R. R. E. Salomaa, and M. Shoucri, Phys. Rev. E 49, 5656 (1994).

${ }^{8}$ D. Umstadter, S.-Y. Chen, A. Maksimchuk, G. Mourou, and R. Wagner, Science 273, 472 (1996).

${ }^{9}$ C. I. Moore, A. Ting, K. Krushelnick et al., Phys. Rev. Lett. 79, 3909 (1997).

${ }^{10}$ S. V. Bulanov, F. Pegoraro, A. M. Pukhov, and A. S. Sakharov, Phys. Rev. Lett. 78, 4205 (1997).

${ }^{11}$ D. Umstadter, J. K. Kim, and E. Dodd, Phys. Rev. Lett. 76, 2073 (1996).

${ }^{12}$ E. Esarey, R. F. Hubbard, W. P. Leemans, A. Ting, and P. Sprangle, Phys. Rev. Lett. 79, 2682 (1997).

${ }^{13}$ A. Modena, Z. Najmudin, A. E. Dangor et al., Nature (London) 377, 606 (1995).

${ }^{14}$ R. Wagner, S.-Y. Chen, A. Maksimchuk, and D. Umstadter, Phys. Rev. Lett. 78, 3125 (1997).

${ }^{15}$ G. Malka, J. Fuchs, F. Amiranoff et al., Phys. Rev. Lett. 79, 2053 (1997).

${ }^{16}$ D. Gordon, K. C. Tzeng, C. E. Clayton, A. E. Dangor, V. Malka, K. A. Marsh, A. Modena, and W. B. Mora, Phys. Rev. Lett. 80, 2133 (1998).

${ }^{17}$ C. D. Decker, W. B. Mori, and T. Katsouleas, Phys. Rev. E 50, R3338 (1994).
${ }^{18}$ N. E. Andreev, L. M. Gorbunov, and S. V. Kuznetsov, IEEE Trans. Plasma Sci. PS-24, 448 (1996).

${ }^{19}$ K.-C. Tzeng, W. B. Mori, and T. Katsouleas, Phys. Rev. Lett. 79, 5258 (1997).

${ }^{20}$ S. Wilks, T. Katsouleas, J. M. Dawson, P. Chen, and J. J. Su, IEEE Trans. Plasma Sci. PS-15, 210 (1987).

${ }^{21}$ P. Mora, J. Appl. Phys. 71, 2087 (1992).

${ }^{22}$ P. Mora and F. Amiranoff, J. Appl. Phys. 66, 3476 (1989).

${ }^{23}$ E. Esarey and M. Pilloff, Phys. Plasmas 2, 1432 (1995).

${ }^{24}$ A. I. Akheizer and R. V. Polovin, Sov. Phys. JETP 3, 696 (1956).

${ }^{25}$ J. B. Rosenzweig, Phys. Rev. A 38, 3634 (1988).

${ }^{26}$ T. Katsouleas and W. B. Mori, Phys. Rev. Lett. 61, 90 (1988).

${ }^{27}$ J. Dawson, Phys. Fluids 4, 869 (1961).

${ }^{28}$ T. O’Neil, Phys. Fluids 8, 2255 (1965).

${ }^{29}$ G. Brodin, Phys. Rev. Lett. 78, 1263 (1997).

${ }^{30}$ L. Landau, J. Phys. (Moscow) 10, 25 (1946).

${ }^{31}$ S.-Y. Chen, G. S. Sarkisov, A. Maksimchuk, R. Wagner, and D. Umstadter, Phys. Rev. Lett. 80, 2610 (1998).

${ }^{32}$ A. Ting, E. Esarey, and P. Sprangle, Phys. Fluids B 2, 1390 (1990).

${ }^{33}$ S. V. Bulanov, I. N. Inovenkov, V. I. Kirsanov, N. M. Naumova, and A. S. Sakharov, Phys. Fluids B 4, 1935 (1992).

${ }^{34}$ D. Teychenné, G. Bonnaud, and J.-L. Bobin, Phys. Plasmas 1, 1771 (1994).

${ }^{35}$ T. Katsouleas, C. Joshi, J. M. Dawson, F. F. Chen, C. Clayton, W. B. Mori, C. Darrow, and D. Umstadter, in "Laser acceleration of particles," Vol. 130 of Proceedings of the American Institute of Physics Conference, edited by C. Joshi and T. Katsouleas (American Institute of Physics, New York, 1985), p. 63.

${ }^{36} \mathrm{~W}$. Horton and T. Tajima, Phys. Rev. A 34, 4110 (1986).

${ }^{37}$ K.-C. Tzeng, W. B. Mori, and C. D. Decker, Phys. Rev. Lett. 76, 3332 (1996).

${ }^{38}$ S. P. Le Blanc, M. C. Downer, R. Wagner, S.-Y. Chen, A. Maksimchuk, G. Mourou, and D. Umstadter, Phys. Rev. Lett. 77, 5381 (1996).

${ }^{39}$ X. J. Wang, M. Babzien, K. Batchelor et al., Nucl. Instrum. Methods Phys. Res. A 375, 82 (1996).

${ }^{40}$ C. Pellegrini, J. Rosenzweig, G. Travish et al., Nucl. Instrum. Methods Phys. Res. A 341, 326 (1994).

${ }^{41}$ R. L. Sheffield, R. H. Austin, K. C. D. Chan et al., Nucl. Instrum. Methods Phys. Res. A 341, 371 (1994).

${ }^{42}$ W. H. H. Panofsky and W. A. Wenzel, Rev. Sci. Instrum. 27, 976 (1956).

${ }^{43}$ P. Mora and T. M. Antonsen, Jr., Phys. Rev. E 53, R2068 (1996).

${ }^{44}$ G. Malka and J. L. Miquel, Phys. Rev. Lett. 77, 75 (1996).

${ }^{45}$ C. D. Decker and W. B. Mori, Phys. Rev. Lett. 72, 490 (1994).

${ }^{46}$ E. Weibel, Phys. Rev. Lett. 2, 83 (1959).

${ }^{47}$ C. F. Driscoll and K. S. Fine, Phys. Fluids B 2, 1359 (1990).

${ }^{48}$ L. Gorbunov, P. Mora, and T. M. Antonsen, Jr., Phys. Rev. Lett. 76, 2495 (1996). 